\title{
Predicting Successful Conservative Surgery after Neoadjuvant Chemotherapy in Hormone Receptor-Positive, HER2-Negative Breast
} Cancer

\author{
Chang Seok Ko, Kyu Min Kim, Jong Won Lee, Han Shin Lee, Sae Byul Lee, Guiyun Sohn, Jisun Kim, Hee Jeong Kim, \\ Il Yong Chung, Beom Seok Ko, Byung Ho Son, Seung Do Ahn ${ }^{1}$, Sung-Bae Kim², Hak Hee Kim³ , Sei Hyun Ahn \\ Division of Breast Surgery, Department of Surgery, Departments of ${ }^{1}$ Radiation Oncology, ${ }^{2}$ Oncology, and ${ }^{3}$ Radiology, Asan Medical Center, University \\ of Ulsan College of Medicine, Seoul, Korea
}

Purpose: This study aimed to determine whether clinicopathological factors are potentially associated with successful breast-conserving surgery (BCS) after neoadjuvant chemotherapy (NAC) and develop a nomogram for predicting successful BCS candidates, focusing on those who are diagnosed with hormone receptor (HR)-positive, human epidermal growth factor receptor 2 (HER2)-negative tumors during the preNAC period. Methods: The training cohort included 239 patients with an HR-positive, HER2-negative tumor $(\geq 3 \mathrm{~cm})$, and all of these patients had received NAC. Patients were excluded if they met any of the following criteria: diffuse, suspicious, malignant microcalcification (extent $>4 \mathrm{~cm}$ ); multicentric or multifocal breast cancer; inflammatory breast cancer; distant metastases at the time of diagnosis; excisional biopsy prior to NAC; and bilateral breast cancer. Multivariate logistic regression analysis was conducted to evaluate the possible predictors of BCS eligibility after NAC, and the regression model was used to develop the predicting nomogram. This nomogram was built using the training cohort $(n=239)$ and was later validated with an independent validation cohort $(n=123)$. Results: Small tumor size $(p<0.001)$ at initial diagnosis, long distance from the nipple $(p=0.002)$, high body mass index $(p=0.001)$, and weak positivity for progesterone receptor $(p=0.037)$ were found to be four independent predictors of an increased probability of BCS after NAC; further, these variables were used as covariates in developing the nomogram. For the training and validation cohorts, the areas under the receiver operating characteristic curve were 0.833 and 0.786 , respectively; these values demonstrate the potential predictive power of this nomogram. Conclusion: This study established a new nomogram to predict successful BCS in patients with HR-positive, HER2-negative breast cancer. Given that chemotherapy is an option with unreliable outcomes for this subtype, this nomogram may be used to select patients for NAC followed by successful BCS.

Key Words: Breast neoplasms, Neoadjuvant therapy, Nomograms, Segmental mastectomy

\section{INTRODUCTION}

From a surgeon's perspective, treating breast cancer patients with neoadjuvant chemotherapy (NAC) provides two major clinical advantages. Firstly, it permits control of locally advanced or inflammatory tumors that cannot be resolved with surgical therapy alone; secondly, it enables breast-conserving surgery (BCS) by reducing the tumor size. There exists no disagreement about the role of NAC as the standard of care in locally advanced or inflammatory breast cancer [1-4]; however,

\section{Correspondence: Jong Won Lee}

Division of Breast Surgery, Department of Surgery, Asan Medical Center,

University of Ulsan College of Medicine, 88 Olympic-ro 43-gil, Songpa-gu, Seoul 05505, Korea

Tel: +82-2-3010-5603, Fax: +82-2-474-9027, E-mail: jjjongwr@hanmail.net

This study was supported by a grant (Elimination of Cancer Project Fund) from the Asan Cancer Institute of Asan Medical Center, Seoul, Korea.

Received: Jun 13, 2018 Revised: Aug 3, 2018 Accepted: Oct 3, 2018 when considering NAC in early breast cancer treatment to reduce the tumor size, there are not many predictors known for successful conservative surgery [3-5].

Tumor biology, that is, subtypes practically determined by estrogen receptor (ER), progesterone receptor (PR), and human epidermal growth factor receptor 2 (HER2) are strong predictors of successful, less invasive surgical approaches after NAC. In a prospective multicenter clinical trial, patients with triple-negative and HER2-positive tumors were found to have high rates of BCS after NAC (46.8\% and $43 \%$, respectively) than those with hormone receptor (HR)-positive, HER2-negative tumors (34.5\%) [5]. Based on the nomograms predicting whether a patient would be eligible for BCS after NAC $[3,4]$ and data from the U.S. National Cancer Database [6], ER negativity is considered to be one of the key factors associated with a high probability of successful breast conservation. Accordingly, surgeons expect that 
large HR-positive and HER2-negative tumors will respond poorly to NAC, subsequently limiting the eligibility for BCS in these patients. Despite the clinical need for more detailed studies with this HR-positive, HER2-negative subtype, very few reliable factors have been identified so far.

It is challenging for surgeons to identify the eligible BCS candidates within the HR-positive and HER2-negative subtype. However, with the assistance of novel genomic assays, careful considerations can be made about treatment strategies for HR-positive, HER2-negative cancer. Based on chemosensitivity and breast cancer mortality, adjuvant chemotherapy should be avoided in selected patients, including women with limited node metastasis [7-9]. For patients representing this subtype and fulfilling the criteria for BCS (except for tumor size), the decision about the appropriateness of NAC can become even more challenging for surgeons.

The first aim of this study was to determine whether the clinicopathologic factors are potentially associated with successful BCS following NAC. Secondly, we aimed to develop a nomogram predicting successful BCS candidates, especially involving those who were diagnosed with HR-positive, HER2-negative tumors during the pre-NAC period.

\section{METHODS}

\section{Study population}

The anonymized database of the Asan Medical Center was reviewed to identify stage II/III HR-positive, HER2-negative breast cancer patients who had received NAC followed by surgery between February 2008 and December 2014. This study included patients with an HR-positive, HER2-negative tumor ( $\geq 3 \mathrm{~cm}$ in size) and had received NAC. We excluded the cases with multicentric tumors or diffuse, suspicious, malignant microcalcification because these factors are generally expected to remain unresolved despite NAC and rarely influence the possibility of conversion from BCS-ineligible to BCS-eligible after NAC. Therefore, patients were excluded if they had diffuse suspicious malignant microcalcification (extent $>4 \mathrm{~cm}$ ) and multicentric or multifocal breast cancer. Patients with the following characteristics were also excluded: inflammatory breast cancer, distant metastases at the time of diagnosis, excisional biopsy prior to NAC, and bilateral breast cancer. Finally, a total of 239 patients were included in our study, and we retrospectively collected information regarding basic demographics as well as clinical, radiological, and pathological factors. After building a nomogram with this cohort, an independent cohort of patients $(n=123)$ was employed to validate the results of the nomogram. The selection of all the patients in the validation set was based on the same inclusion and exclusion criteria as the training set. Between January 2015 and August 2017, these patients also received NAC followed by surgery at the same institution.

Pre-NAC factors, such as tumor size before NAC and the distance between the nipple and the lesion, were examined by breast magnetic resonance imaging. Mammography results showing suspicious microcalcification (American College of Radiology Breast Imaging-Reporting and Data System C4a or higher) around the lesion were considered positive for calcification. For all clinically suspicious cases, fine needle aspiration cytology was used to confirm the presence of lymph node metastasis. The ER and PR levels were assessed with core needle biopsy, and samples were considered positive if there were at least $1 \%$ positively stained tumor nuclei as detected by immunohistochemistry. Additionally, the ER and PR Allred scores were calculated for these samples [10], and weak, intermediate, and strong positive reactions were indicated by the scores $3-4,5-6$, and $7-8$, respectively. The HR-positive group represented patients with ER- and/or PR-positive tumors. HER2 positivity was measured by immunohistochemistry (a score of 3+) or gene amplification quantified by fluorescence in situ hybridization or silver in situ hybridization [11]. Most patients were treated preoperatively with anthracycline- and/or taxane-based chemotherapy. This study was approved by our Institutional Review Board, and informed consent was waived for this retrospective analysis (IRB number: 2017-1341).

\section{Statistical analysis}

Correlations between the definitive surgery type and several categorical variables were evaluated using the chi-square test. For continuous variables such as age, body mass index (BMI), initial tumor size, and distance from the nipple, a t-test was used for comparing the means of BCS and total mastectomy groups. Multivariate logistic regression analysis was conducted to evaluate possible predictors for BCS eligibility after NAC. A stepwise variable selection was performed to include significant independent variables in the model. Subsequently, we developed a regression model-derived nomogram 
by employing a training cohort $(\mathrm{n}=239)$ and further validated with another independent cohort $(\mathrm{n}=123)$. The model performance was assessed with respect to discrimination, which was quantified with the concordance index, identical to the area under the receiver operating characteristics (ROC) curve. Unless stated otherwise, the data are presented as the mean \pm standard deviation, and the cutoff for statistical significance is set at $p<0.05$. All statistical analyses were performed using SPSS version 18.0 (SPSS Inc., Chicago, USA), STATA version 13 (StataCorp., College Station, USA), and open source R version 3.4.

\section{RESULTS}

The age of the entire training cohort $(\mathrm{n}=239$ patients) was $45.2 \pm 9.6$ years (range, 23-72 years), and the initial tumor size was $5.3 \pm 1.7 \mathrm{~cm}$ (range, $3.1-11.8 \mathrm{~cm}$ ). There were 141 patients (59.0\%) detected with positive nodes during preoperative diagnosis, and 15 patients (6.3\%) achieved a complete pathological response (pCR) to NAC. For NAC regimens, four cycles of adriamycin along with cyclophosphamide every 3 weeks (AC) or four cycles of adriamycin combined with cyclophosphamide every 3 weeks followed by four cycles of docetaxel every 3 weeks (AC-D) was given to $86(36.0 \%)$ and $116(48.5 \%)$ patients, respectively. The details of the patient characteristics are provided in Table 1.

Table 2 summarizes the baseline characteristics of patients undergoing BCS and total mastectomy. BMI, initial tumor size, clinical nodal status, histologic grade, ER status, the regimen of chemotherapy, malignant calcification, and distance from the nipple were significantly different between the two groups.

The results of the multivariate logistic regression analysis (Table 3) demonstrated that the four variables including initial tumor size $(p<0.001)$, distance from the nipple $(p=0.002)$, BMI $(p=0.001)$, and $\operatorname{PR}(p=0.037)$ were independent predictors of an increased probability of BCS after NAC. These variables (small tumor size at the initial diagnosis, long distance from the nipple, high BMI, and weak PR positivity) were related to successful BCS after NAC. Thus, these predictors were used to develop a predicting nomogram (Figure 1). Each predictor and corresponding point are presented in Table 4. Using these four predictors, we calculated total scores and assigned probabilities of successful BCS for each patient either by directly reading displayed values in Table 4 or via approximate graphical computation
Table 1. Baseline characteristics of 239 patients with HR-positive, HER2-negative breast cancer

\begin{tabular}{|c|c|}
\hline Characteristic & №. (\%) \\
\hline Age $(y r)^{*}$ & $45.2 \pm 9.6(23-72)$ \\
\hline$<50$ & $162(67.8)$ \\
\hline$\geq 50$ & $77(32.2)$ \\
\hline $\operatorname{BMI}\left(\mathrm{kg} / \mathrm{m}^{2}\right)^{*}$ & $24.4 \pm 3.7(16.9-41.2)$ \\
\hline Underweight (15-19.9) & $20(8.4)$ \\
\hline Normal weight (20-24.9) & $131(54.8)$ \\
\hline Overweight ( $\geq 25$ ) & $88(36.8)$ \\
\hline Initial tumor size $(\mathrm{cm})^{*}$ & $5.3 \pm 1.7(3.1-11.8)$ \\
\hline \multicolumn{2}{|l|}{ Clinical nodal status } \\
\hline Negative metastasis & $98(41.0)$ \\
\hline Positive metastasis & $141(59.0)$ \\
\hline \multicolumn{2}{|l|}{ Calcification } \\
\hline Yes & $140(58.6)$ \\
\hline No & $99(41.4)$ \\
\hline \multicolumn{2}{|l|}{ Histologic type } \\
\hline Invasive ductal carcinoma & $225(94.1)$ \\
\hline Invasive lobular carcinoma & $11(4.6)$ \\
\hline Others & $3(1.3)$ \\
\hline \multicolumn{2}{|l|}{ Histologic grade } \\
\hline Grade $1 / 2$ & $192(80.3)$ \\
\hline Grade 3 & $42(17.6)$ \\
\hline Unknown & $5(2.1)$ \\
\hline \multicolumn{2}{|l|}{ Estrogen receptor status } \\
\hline Strong & $217(90.8)$ \\
\hline Intermediate/weak/negative & $22(9.2)$ \\
\hline \multicolumn{2}{|l|}{ Progesterone receptor status } \\
\hline Strong & $158(66.1)$ \\
\hline Intermediate/weak/negative & $81(33.9)$ \\
\hline \multicolumn{2}{|l|}{ Ki-67 status (\%) } \\
\hline$<20$ & $69(28.9)$ \\
\hline$\geq 20$ & $144(60.1)$ \\
\hline Unknown & $26(11.0)$ \\
\hline \multicolumn{2}{|l|}{ Chemotherapy regimen } \\
\hline $\mathrm{AC \# 4}$ & $86(36.0)$ \\
\hline AC\#4 followed by D\#4 & $116(48.5)$ \\
\hline Others & $37(15.5)$ \\
\hline \multicolumn{2}{|l|}{ Type of surgery } \\
\hline Breast-conserving surgery & $108(45.2)$ \\
\hline Total mastectomy & $131(54.8)$ \\
\hline \multicolumn{2}{|l|}{$\mathrm{pCR}$} \\
\hline Yes & $15(6.3)$ \\
\hline No & 224 (93.7) \\
\hline
\end{tabular}

$\mathrm{HR}=$ hormone receptor; $\mathrm{HER} 2$ = human epidermal growth factor receptor 2; $\mathrm{BMI}=$ body mass index; $\mathrm{AC}=$ adriamycin+cyclophosphamide; $\mathrm{D}=$ docetaxel; $\mathrm{pCR}=$ pathologic complete response. *Mean \pm SD (range).

as shown in Figure 1.

Figure 2 shows the ROC curves that were used to determine the discrimination power of the nomogram. These curves were applied to the training dataset ( $\mathrm{n}=239$ patients) with known values or status of 
Table 2. Tumor/patient characteristics according to type of definitive surgery

\begin{tabular}{|c|c|c|c|}
\hline \multirow[b]{2}{*}{ Characteristic } & \multicolumn{2}{|c|}{ Definitive surgery group } & \multirow[b]{2}{*}{$p$-value } \\
\hline & $\begin{array}{c}\text { BCS }(n=108) \\
\text { No. }(\%)\end{array}$ & $\begin{array}{c}\text { TM }(n=131) \\
\text { No. }(\%)\end{array}$ & \\
\hline Age $(y r)^{*}$ & $45.0 \pm 9.6(23-70)$ & $45.8 \pm 9.7(27-72)$ & 0.507 \\
\hline$<50$ & $76(46.9)$ & $86(53.1)$ & \\
\hline$\geq 50$ & $32(41.6)$ & $45(58.4)$ & \\
\hline BMI $\left(\mathrm{kg} / \mathrm{m}^{2}\right)^{*}$ & $25.4 \pm 3.9(18.4-41.1)$ & $23.6 \pm 3.3(16.9-34.0)$ & $<0.001$ \\
\hline Underweight (15-19.9) & $5(25.0)$ & $15(75.0)$ & \\
\hline Normal weight (20-24.9) & $54(41.2)$ & $77(58.8)$ & \\
\hline Overweight $(\geq 25)$ & $49(55.7)$ & $39(44.3)$ & \\
\hline Initial tumor size $(\mathrm{cm})^{*}$ & $4.4 \pm 1.3(3.1-8.6)$ & $6.0 \pm 1.7(3.1-11.8)$ & $<0.001$ \\
\hline Distance from nipple $(\mathrm{cm})^{*}$ & $3.4 \pm 2.0(0-9.2)$ & $1.8 \pm 1.8(0-7.5)$ & $<0.001$ \\
\hline Clinical nodal status & & & $<0.001$ \\
\hline Negative metastasis & $59(59.0)$ & $41(41.0)$ & \\
\hline Positive metastasis & $49(35.3)$ & $90(64.7)$ & \\
\hline Histologic type & & & 0.207 \\
\hline Invasive ductal carcinoma & $105(46.7)$ & $120(53.3)$ & \\
\hline Invasive lobular carcinoma & $3(27.3)$ & $8(72.7)$ & \\
\hline Others & 0 & 3 & \\
\hline Histologic grade & & & 0.003 \\
\hline Grade $1 / 2$ & $80(41.7)$ & $112(58.3)$ & \\
\hline Grade 3 & $28(66.7)$ & $14(33.3)$ & \\
\hline Unknown & 0 & 5 & \\
\hline Estrogen receptor status & & & 0.027 \\
\hline Strong & $80(41.7)$ & $112(58.3)$ & \\
\hline Intermediate/negative/weak & $28(59.6)$ & $19(40.4)$ & \\
\hline Progesterone receptor status & & & 0.233 \\
\hline Strong & $42(40.8)$ & $61(59.2)$ & \\
\hline Intermediate/negative/weak & $66(48.5)$ & $70(51.5)$ & \\
\hline Ki-67 status (\%) & & & 0.110 \\
\hline$<20$ & $26(37.7)$ & $43(62.3)$ & \\
\hline$\geq 20$ & $71(49.3)$ & $73(50.7)$ & \\
\hline Unknown & 11 & 15 & \\
\hline Chemotherapy regimen & & & $<0.001$ \\
\hline $\mathrm{AC \# 4}$ & $46(53.5)$ & $40(46.5)$ & \\
\hline AC\#4 followed by D\#4 & $46(39.7)$ & $70(60.3)$ & \\
\hline Others & $16(43.2)$ & $21(56.8)$ & \\
\hline Calcification & & & 0.015 \\
\hline Yes & $54(38.6)$ & $86(61.4)$ & \\
\hline No & $54(54.5)$ & $45(45.5)$ & \\
\hline $\mathrm{pCR}$ & & & 0.024 \\
\hline Yes & $11(73.3)$ & $4(26.7)$ & \\
\hline No & $97(43.3)$ & $127(56.7)$ & \\
\hline
\end{tabular}

$\mathrm{BCS}=$ breast-conserving surgery; $\mathrm{TM}=$ total mastectomy; $\mathrm{BMI}=$ body mass index; $\mathrm{AC}=$ adriamycin+cyclophosphamide; $\mathrm{D}=$ docetaxel; $\mathrm{pCR}=$ pathologic complete response.

${ }^{*}$ Mean \pm SD (range).

Table 3. Multivariate logistic regression analysis of clinicopathologic variables for predicting BCS eligibility after NAC

\begin{tabular}{lccc}
\hline Variable & Odds ratio & $95 \% \mathrm{Cl}$ & $p$-value \\
\hline Initial tumor size $(\mathrm{cm})$ & 0.51 & $0.40-0.66$ & $<0.001$ \\
Distance from nipple $(\mathrm{cm})$ & 1.33 & $1.11-1.60$ & 0.002 \\
BMI $\left(\mathrm{kg} / \mathrm{m}^{2}\right)$ & 1.18 & $1.07-1.31$ & 0.001 \\
PR (strong vs. intermediate/weak/negative) & 0.48 & $0.23-0.96$ & 0.037
\end{tabular}

$\mathrm{BCS}=$ breast-conserving surgery; $\mathrm{NAC}=$ neoadjuvant chemotherapy; $\mathrm{Cl}=$ confidence interval; $\mathrm{BMI}=$ body mass index; $\mathrm{PR}=$ progesterone receptor. 
Point

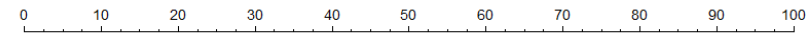

Distance from nipple $(\mathrm{cm})$

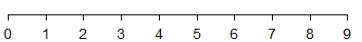

$\operatorname{BMI}\left(\mathrm{kg} / \mathrm{m}^{2}\right)$

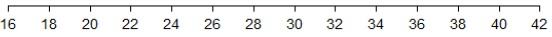

Initial tumor size $(\mathrm{cm})$

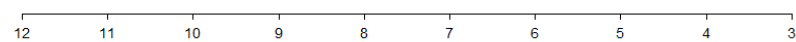

$\mathrm{PR}$ (1, strong; 0, non-strong)

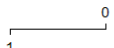

Total points

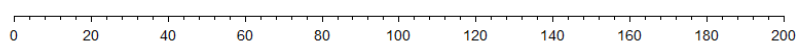

BCS success possibility

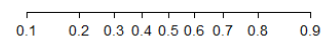

Figure 1. Nomogram predicting successful breast-conserving surgery (BCS) candidates especially those diagnosed with the hormone receptor-positive, human epidermal growth factor receptor 2-negative tumors during the pre-neoadjuvant chemotherapy period.

$\mathrm{BMl}=$ body mass index; $\mathrm{PR}=$ progesterone receptor.

Table 4. Corresponding points for four predictors and final BCS success probability

\begin{tabular}{|c|c|c|c|c|c|c|c|c|c|}
\hline \multicolumn{8}{|c|}{ Predictors } & \multirow[b]{2}{*}{ Total points } & \multirow{2}{*}{$\begin{array}{l}\text { BCS success } \\
\text { probability }\end{array}$} \\
\hline $\begin{array}{c}\text { Distance from } \\
\text { nipple }(\mathrm{cm})\end{array}$ & Point & $\begin{array}{c}\text { BMI } \\
\left(\mathrm{kg} / \mathrm{m}^{2}\right)\end{array}$ & Point & $\begin{array}{l}\text { Initial tumor } \\
\text { size }(\mathrm{cm})\end{array}$ & Point & PR & Point & & \\
\hline 0 & 0 & 17 & 0 & 3 & 100 & 0 (strong) & 12 & 68 & 0.05 \\
\hline 0.5 & 2 & 18 & 3 & 3.5 & 94 & $\begin{array}{l}1 \text { (intermediate/ } \\
\text { weak/negative) }\end{array}$ & 0 & 81 & 0.1 \\
\hline 1 & 5 & 19 & 5 & 4 & 89 & - & - & 89 & 0.15 \\
\hline 1.5 & 7 & 20 & 8 & 4.5 & 83 & - & - & 94 & 0.2 \\
\hline 2 & 10 & 21 & 11 & 5 & 78 & - & - & 99 & 0.25 \\
\hline 2.5 & 12 & 22 & 13 & 5.5 & 72 & - & - & 104 & 0.3 \\
\hline 3 & 15 & 23 & 16 & 6 & 67 & - & - & 107 & 0.35 \\
\hline 3.5 & 17 & 24 & 19 & 6.5 & 61 & - & - & 111 & 0.4 \\
\hline 4 & 20 & 25 & 21 & 7 & 56 & - & - & 114 & 0.45 \\
\hline 4.5 & 22 & 26 & 24 & 7.5 & 50 & - & - & 118 & 0.5 \\
\hline 5 & 25 & 27 & 27 & 8 & 44 & - & - & 121 & 0.55 \\
\hline 5.5 & 27 & 28 & 29 & 8.5 & 39 & - & - & 125 & 0.6 \\
\hline 6 & 29 & 29 & 32 & 9 & 33 & - & - & 128 & 0.65 \\
\hline 6.5 & 32 & 30 & 35 & 9.5 & 28 & - & - & 132 & 0.7 \\
\hline 7 & 34 & 31 & 37 & 10 & 22 & - & - & 136 & 0.75 \\
\hline 7.5 & 37 & 32 & 40 & 10.5 & 17 & - & - & 141 & 0.8 \\
\hline 8 & 39 & 33 & 43 & 11 & 11 & - & - & 147 & 0.85 \\
\hline 8.5 & 42 & 34 & 45 & 11.5 & 6 & - & - & 155 & 0.9 \\
\hline- & - & 35 & 48 & 12 & 0 & - & - & 167 & 0.95 \\
\hline- & - & 36 & 50 & - & - & - & - & - & - \\
\hline- & - & 37 & 53 & - & - & - & - & - & - \\
\hline- & - & 38 & 56 & - & - & - & - & - & - \\
\hline- & - & 39 & 58 & - & - & - & - & - & - \\
\hline- & - & 40 & 61 & - & - & - & - & - & - \\
\hline- & - & 41 & 64 & - & - & - & - & - & - \\
\hline
\end{tabular}

$\mathrm{BCS}=$ breast-conserving surgery $\mathrm{BMI}=$ body mass index $\mathrm{PR}=$ progesterone receptor. 

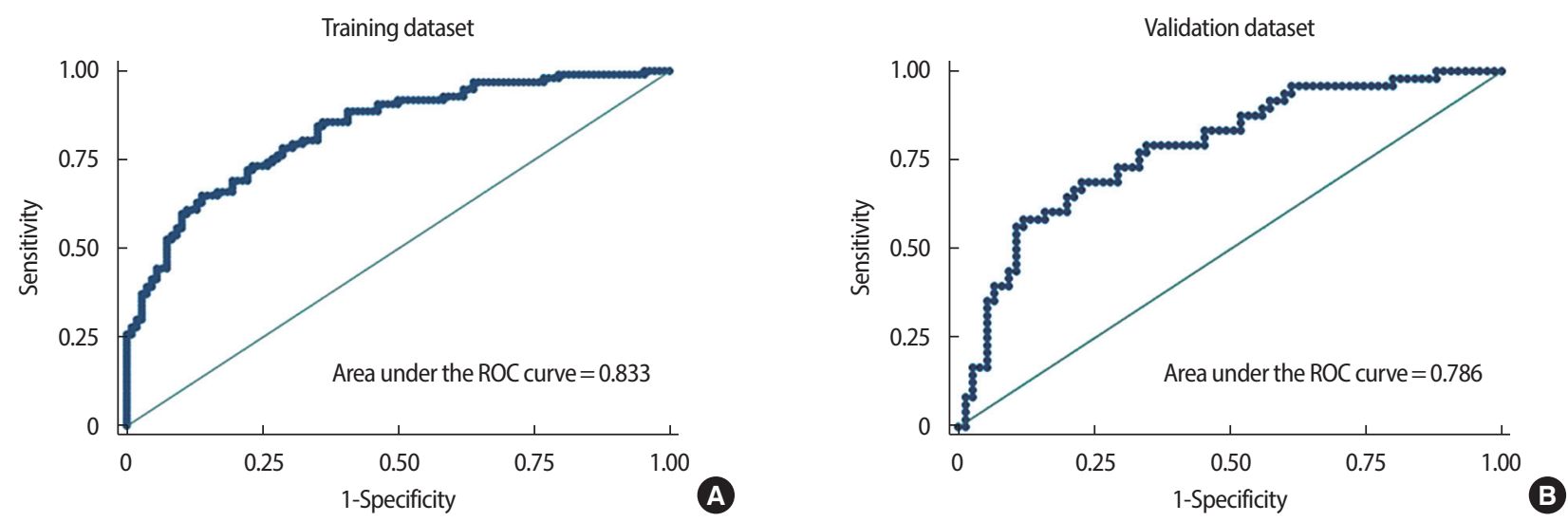

Figure 2. Receiver operating characteristic (ROC) curves of the constructed nomogram predicting successful breast-conserving surgery candidates in the training (A) and validation (B) datasets.

all four predictors, and they were later applied to the independent validation dataset ( $\mathrm{n}=123$ patients). The areas under the ROC curve (AUCs) were 0.833 and 0.786 , respectively, and these values demonstrated a strong predictive power of this nomogram.

\section{DISCUSSION}

In this study, we identified four factors (i.e., small initial tumor size, long distance from the nipple, high BMI, and weak PR positivity) as predictors of successful BCS. Our nomogram holds promising discriminatory power in identifying HR-positive, HER2-negative breast cancer patients who have received NAC.

Although many studies have investigated pCR rates according to breast cancer subtype, very few have investigated its impact on the surgical procedure. Boughey et al. [5] reported the rates of BCS for patients participating in the American College of Surgeons Oncology Group (ACOSOG) Z1071 trial. They found that the rate of BCS was higher in patients with triple-negative and HER2-positive breast cancer (46.8\% and 43\%, respectively) than in those with HR-positive, HER2-negative cancer $(34.5 \%, p=0.019)$. The MD Anderson Cancer Center (MDACC) nomogram [3] also showed that ER negativity is independently associated with eligibility for BCS. For surgeons, however, more information on predicting successful BCS candidates in this HR-positive, HER2-negative subtype is critical for counseling patients, particularly if BCS conversion is the only purpose of NAC. Compared with previous studies involving patients with all types of breast cancer and identifying the subtype as a key predictor [3-
6,12,13], the current study has valuable implications because our nomogram can be specifically applied to patients with HR-positive, HER2-negative cancer, which is believed to be less responsive to NAC and has a low probability of successful BCS.

At the initial diagnosis, the appearance of a small tumor is intuitively understood as a predictor of successful BCS. This enables surgeons to make a decision favoring BCS; further, tumor size has been consistently reported as being significant in other nomograms [3,4]. Long distance from the nipple enhances the ability to conserve the nipple-areolar complex (NAC) and also encourages surgeons to recommend BCS. Undoubtedly, central lumpectomy with resection of NAC may be chosen as a safe and cosmetic surgical option; however, this decision depends on a surgeon's preference $[14,15]$. Therefore, it is recommended that at institutes where central lumpectomy is more frequently performed, the significance of distance of tumor from the nipple should be cautiously re-examined. Patients with high BMI have increased risks of receiving BCS, possibly because BMI is significantly associated with breast volume; further, it is known that the breast volume is two-three times greater in overweight and obese women (with high BMI) than in women with normal BMI [16]. Contrary to our results, in the previously presented Seoul National University Hospital (SNUH) nomogram, Kim et al. [4] reported that the breast volume was not a predictive factor for BCS. These results may have been influenced by either the two-dimensional, mammogram-based method used in this study [17] or difference in their approach to analyze the study cohorts. The SNUH nomogram included all subtypes while our nomogram exclusively focused on patients with 
HR-positive, HER2-negative subtype. Another possible explanation is that BMI can affect pCR [18]. However, our analyses did not reveal any relationship between BMI and pathological (complete or partial) response in the patient cohort (data not shown).

From a surgeon's perspective, it is important to identify the clinical factors that may impact the outcomes of surgical procedures, not the response to NAC. At the beginning of the study, patients were excluded if they showed diffuse suspicious malignant microcalcification (extent $>4 \mathrm{~cm}$ ), multicentric or multifocal breast cancer because these factors are most likely not resolved even after NAC. Further, they rarely influence the possibility of conversion from BCS-ineligible to BCS-eligible following NAC. Our nomogram does not include factors like microcalcification (included in the SNUH nomogram) and multicentricity (included both in the MDACC and SNUH nomograms). These factors may explain the discrepancies observed in our results. Another striking finding of this study is that our AUC values $(0.833$ and 0.786 ) are superior to those presented in the MDACC (AUC= 0.790 and 0.710$)$ and SNUH $(\mathrm{AUC}=0.800)$ nomograms. Furthermore, it should be mentioned that while the MDACC and SNUH nomograms included all subtypes, our nomogram was established from a subtype supposedly less responsive to NAC.

Additionally, further analyses were conducted to investigate the tumor size shrinkage prediction as well as the successful BCS prediction for this subtype. However, the results of the multivariate logistic regression analyses demonstrated that neither residual tumor size of $\leq 3$ $\mathrm{cm}$ nor partial response was successfully identified by the predictors (data not shown); this may be due to the poor responsiveness of this subtype.

There are several limitations of this study worth noting. This study was performed using retrospective data, and treatment was not assigned in a randomized fashion. In addition, there might be a bias in predicting BCS because patients' preferences for BCS are not reflected in this study, and patients who underwent total mastectomy followed by immediate reconstruction are also included in this cohort. In future, a detailed and planned survey of the surgery type in a prospective setting may provide a nomogram with improved predictive capabilities.

In conclusion, this study establishes a new nomogram to predict successful BCS in patients with HR-positive, HER2-negative breast cancer. Given that chemotherapeutic response is unpredictable for this subtype of breast cancer, our nomogram can be useful in selecting the patients who will benefit from NAC followed by successful BCS.

\section{CONFLICT OF INTEREST}

The authors declare that they have no competing interests.

\section{REFERENCES}

1. Sinclair S, Swain SM. Primary systemic chemotherapy for inflammatory breast cancer. Cancer 2010;116(11 Suppl):2821-8.

2. Specht J, Gralow JR. Neoadjuvant chemotherapy for locally advanced breast cancer. Semin Radiat Oncol 2009;19:222-8.

3. Rouzier R, Pusztai L, Garbay JR, Delaloge S, Hunt KK, Hortobagyi GN, et al. Development and validation of nomograms for predicting residual tumor size and the probability of successful conservative surgery with neoadjuvant chemotherapy for breast cancer. Cancer 2006;107:1459-66.

4. Kim MK, Han W, Moon HG, Ahn SK, Kim J, Lee JW, et al. Nomogram for predicting breast conservation after neoadjuvant chemotherapy. Cancer Res Treat 2015;47:197-207.

5. Boughey JC, McCall LM, Ballman KV, Mittendorf EA, Ahrendt GM, Wilke LG, et al. Tumor biology correlates with rates of breast-conserving surgery and pathologic complete response after neoadjuvant chemotherapy for breast cancer: findings from the ACOSOG Z1071 (Alliance) Prospective Multicenter Clinical Trial. Ann Surg 2014;260:608-14.

6. Killelea BK, Yang VQ, Mougalian S, Horowitz NR, Pusztai L, Chagpar $\mathrm{AB}$, et al. Neoadjuvant chemotherapy for breast cancer increases the rate of breast conservation: results from the National Cancer Database. J Am Coll Surg 2015;220:1063-9.

7. Albain KS, Barlow WE, Shak S, Hortobagyi GN, Livingston RB, Yeh IT, et al. Prognostic and predictive value of the 21 -gene recurrence score assay in postmenopausal women with node-positive, oestrogen-receptor-positive breast cancer on chemotherapy: a retrospective analysis of a randomised trial. Lancet Oncol 2010;11:55-65.

8. Martin M, Brase JC, Calvo L, Krappmann K, Ruiz-Borrego M, Fisch $\mathrm{K}$, et al. Clinical validation of the EndoPredict test in node-positive, chemotherapy-treated ER+/HER2- breast cancer 
patients: results from the GEICAM 9906 trial. Breast Cancer Res 2014;16:R38.

9. Cardoso F, van't Veer LJ, Bogaerts J, Slaets L, Viale G, Delaloge S, et al. 70-Gene signature as an aid to treatment decisions in early-stage breast cancer. N Engl J Med 2016;375:717-29.

10. Harvey JM, Clark GM, Osborne CK, Allred DC. Estrogen receptor status by immunohistochemistry is superior to the ligand-binding assay for predicting response to adjuvant endocrine therapy in breast cancer. J Clin Oncol 1999;17:1474-81.

11. Wolff AC, Hammond ME, Hicks DG, Dowsett M, McShane LM, Allison $\mathrm{KH}$, et al. Recommendations for human epidermal growth factor receptor 2 testing in breast cancer: American Society of Clinical Oncology/College of American Pathologists clinical practice guideline update. Arch Pathol Lab Med 2014;138:241-56.

12. Mathieu MC, Mazouni C, Kesty NC, Zhang Y, Scott V, Passeron J, et al. Breast Cancer Index predicts pathological complete response and eligibility for breast conserving surgery in breast cancer patients treated with neoadjuvant chemotherapy. Ann Oncol 2012;23:204652.

13. Hage AN, Capriccioso C, Brennan J, Heiden B, Zheutlin A, Sabel
MS. Impact of neoadjuvant chemotherapy on surgical outcomes among patients with hormone receptor positive breast cancer. J Surg Oncol 2017;116:665-70.

14. Simmons RM, Brennan MB, Christos P, Sckolnick M, Osborne M. Recurrence rates in patients with central or retroareolar breast cancers treated with mastectomy or lumpectomy. Am J Surg 2001;182: 325-9.

15. Lim RJ, Lee KS, Lee SH, Yoon CS, Ko SS, Hur MH, et al. Central lumpectomy with resection of the nipple-areolar complex for retroareolar or central breast cancers. J Korean Surg Soc 2010;79:27580 .

16. Coltman CE, Steele JR, McGhee DE. Breast volume is affected by body mass index but not age. Ergonomics 2017;60:1576-85.

17. Katariya RN, Forrest AP, Gravelle IH. Breast volumes in cancer of the breast. Br J Cancer 1974;29:270-3

18. Warner ET, Ballman KV, Strand C, Boughey JC, Buzdar AU, Carey LA, et al. Impact of race, ethnicity, and BMI on achievement of pathologic complete response following neoadjuvant chemotherapy for breast cancer: a pooled analysis of four prospective Alliance clinical trials (A151426). Breast Cancer Res Treat 2016;159:109-18. 